\title{
Effects of Treatment with Nociceptive Trigeminal Inhibition Splints on Electromyography in Temporomandibular Joint Disorder Patients
}

\author{
Jehad M Kara Boulad ${ }^{1}$, Rabab A Al-Sabbagh ${ }^{2}$, Ahmad S Burhan ${ }^{3}$, Chaza N Kouchaji ${ }^{4}$, Fehmieh R Nawaya ${ }^{5}$
}

\begin{abstract}
Aim: This research aimed at evaluating the effects of the nociceptive trigeminal inhibition splint (NTIS) on electromyography (EMG) for masseter and temporalis muscles in patients with temporomandibular joint disorders (TMDs), and at detecting the discomfort degree originating from this splint.

Materials and methods: The sample consisted of 15 patients having TMDs of muscular origin to be treated by NTIS. The activity degree of masseter and temporalis muscles was measured using the EMG two times: before the treatment and after 6 months. Besides, patients' discomfort was assessed after the start of treatment four times: 1 day, 2 weeks, 1 month, and 6 months.

Results: After the treatment, there was a significant decrease in masseter and temporalis muscles' activity in both right and left sides ( $p<0.001)$. No significant differences were observed in the electrical muscular activity mean change between the masseter muscles $(-43.87 \pm 26.82)$ and the temporalis muscles $(-54.91 \pm 21.16)(p=0.082)$, or between the right muscles $(-51.97 \pm 26.30)$ and the left muscles $(-46.81 \pm 22.90)$ $(p=0.422)$. In addition, the discomfort degree gradually decreased after 2 weeks $(p<0.01)$.

Conclusion: The use of NTIS is associated with reduction in the masseter and temporalis muscles' activity. Also, the patients' discomfort degree from NTIS progressively decreases after 2 weeks of treatment.

Clinical significance: The NTIS is an effective therapeutic approach for patients having TMDs of muscular origin.

Keywords: Electromyography, Masseter, Splints, Temporalis, Temporomandibular joint disorders.

The Journal of Contemporary Dental Practice (2019): 10.5005/jp-journals-10024-2565
\end{abstract}

\section{INTRODUCTION}

The term "temporomandibular joint disorders" (TMDs) was first described in 1934 by James Costen. ${ }^{1}$ In 1983, this term was defined by the American Dental Association as a group of oral disorders. The most important character of which is the pain in the preauricular region, the temporomandibular joint (TMJ), and the masticatory muscles. In addition to deviations or limited motion, there exist occasional joint sounds during movement. ${ }^{2}$ The several causes of TMDs, malocclusions, parafunctional habits like bruxism, stress, or anxiety, may lead to capsule inflammation, muscular pain, spasms, or capsule damage. ${ }^{3}$

There are several treatment approaches for TMDs including surgical and nonsurgical methods. Surgical management comprises of arthrosynthesis or arthroscopy of the TMJ. On the contrary, nonsurgical methods involve education and counseling, occlusal splints, medications, and physiotherapy techniques, such as jaw exercises and electrotherapy. ${ }^{4}$ These conservative methods are the first option for TMJ treatment because of their reversible nature. ${ }^{5}$

Occlusal splints have been used as an important therapeutic tool for the treatment of TMDs for over 100 years. ${ }^{6}$ These splints aim at improving the function of the TMJ, ensuring the stability of functional articular elements during the various jaw motions and promoting the function of the masticatory system. ${ }^{7}$ There are several types of occlusal splints which are mentioned in the medical literature, differing in their design according to their indications and functions. ${ }^{8}$

The nociceptive trigeminal inhibition splint (NTIS) is an intraoral device composed of an anterior bite plane that covers the upper or lower central incisors, and is made of thermoplastic or acrylplastic materials adapted to suit the shape of the central incisors. ${ }^{9}$
1,3 Department of Orthodontics, Faculty of Dentistry, Damascus University, Damascus, Syria

${ }^{2}$ Department of Orthodontics, Faculty of Dentistry, Hama University, Hama, Syria

${ }^{4}$ Department of Pediatric Dentistry, Faculty of Dentistry, Damascus University, Damascus, Syria

${ }^{5}$ Department of Pediatric Dentistry, Faculty of Dentistry, Syrian Private University, Damascus Countryside, Syria

Corresponding Author: Ahmad S Burhan, Department of Orthodontics, Faculty of Dentistry, Damascus University, Syria, Phone: +963944302075, e-mail: dr.burhan-a@hotmail.com

How to cite this article: Boulad JMK, Al-Sabbagh RA, et al. Effects of Treatment with Nociceptive Trigeminal Inhibition Splints on Electromyography in Temporomandibular Joint Disorders Patients. J Contemp Dent Pract 2019;20(5):598-602.

Source of support: $\mathrm{Nil}$

Conflict of interest: None

Ethics approval and consent to participate: The current study was approved by the University of Hama, Dental School, Ethics Committee (No. 310, date 6/1/2015). All participants provided informed consents.

Adjustment of the bite plane is sought by clinicians to ensure that the contact points, in centric occlusion or in lateral movements, are obtained only between the splint and the opposite incisors and not with the opposite canines. This splint was developed to inhibit stimuli and to prevent nonfunctional habits, which lead to the best stability of the articular disc in the upper frontal position. ${ }^{10}$ Moreover, the use of the NTIS can be justified when a reduction of 
jaw closing muscle activity is required as jaw clenching and tooth grinding, or as an emergency device for patients with acute TMJ pain and restricted jaw opening. ${ }^{11}$

Electromyography (EMG) records the electrical potential created when muscular cells are activated by neural or electrical stimulation. These records provide information about muscular contraction, tone, and fatigue. Surface-type EMG has been widely used in the diagnosis of repetitive strain injuries, work-related musculoskeletal disorders, myofascial pain syndrome, fibromyalgia, and rehabilitation training. ${ }^{12}$

Shankland conducted a study to compare the efficacy and safety of full coverage splints and NTIS in reducing the severity and frequency of migraine in 94 patients for 8 weeks. The results revealed that these patients showed a more positive response to the NTIS than the other splints. ${ }^{13}$

There is scarcity of published clinical studies addressing the therapeutic efficacy of NTIS in patients with TMDs. In addition, to the best of our knowledge, no clinical study examined the patients' discomfort degree when treated with it. This study aimed at evaluating the effects of NTIS on the masseter and temporalis muscles' activity using EMG in TMD patients, and detecting patients' discomfort degree to this splint.

\section{Materials and Methods}

The current research is an experimental clinical trial. The research project was approved by the University of Hama, Dental School, Ethics Committee (No. 310, date 6/1/2015). A pilot study ( $N=5$ patients) was conducted to determine the required sample size. The $\mathrm{G}^{*}$ power software (version 3.0.6, Franz Faul, Universitat Kiel, Germany) was used with a paired-sample $t$ test, a selected study power of $90 \%$, a significance level of 0.05 , a number of groups of 1 , and an effect size of 0.983 calculated according to the pilot study. The analysis revealed that 13 patients were required. This number was increased to 15 patients to compensate for the potential dropouts. The participants of the current study ( 10 females and 5 males; age mean $29 \pm 5.6$ years) were recruited from those patients seeking treatment at the Orthodontic Clinic, Hama University. All patients had muscular origin TMDs diagnosed using the clinical manual functional analysis according to Bumann and Lotzmann. ${ }^{14}$ The inclusion criteria were: TMD symptoms for 6 months or more, age is 16 years or older, each patient has minimally 12 teeth in each of the upper and lower jaws, ${ }^{15}$ impaired range of mandibular motion; maximum bite opening is less than $32 \mathrm{~mm},{ }^{16}$ muscular pain in both sides, TMJ pain in both sides, and pain during mandibular movements.

A written informed consent was obtained from each patient. Maxillary NTIS was made from thermoplastic material (Orthocryl, Dentaurum, Germany) covering the incisors. The contact only occurs with the two lower central incisors at closing. At the same time, lower canines should be away from contact with the splint during lateral movements ${ }^{11}$ (Fig. 1). The patients were asked to wear the splints overnight and not to exceed 12 hours a day. ${ }^{9}$

The muscle activity was recorded using an electromyogram (Micromed S.p.A., TOR500 model, Via Giotto, 2-31021 Mogliano Veneto TV, Italy). It is a device measuring the electrical muscular efficiency of the latency difference in the muscle cell membrane of the muscle. Device data are: measuring rate $1,000 \mathrm{~Hz}$ per channel, input impedance $1,000 \mathrm{G} \Omega$ or greater, common mode rejection ratio $110 \mathrm{~dB}$, noise related to input $0.5 \mu \mathrm{V}$, bandwidth $20 \mathrm{~Hz}-20 \mathrm{kHz}$, and signal amplification rate $700 \times$ from each channel.

Considering the fact that the least EMG of masseter and temporalis muscles' activity was found with an intermolar distance of $10 \mathrm{~mm},{ }^{17}$ the EMG activity of both muscles was achieved with a contact area of $10 \mathrm{~mm}$. Surface electrodes were used for recording the muscular activity in the right and left sides. The patients were seated with the horizontal Frankfurt plane parallel to horizon. Prior to electrode placement, the impedance of the skin was reduced by cleaning with alcohol, and a specific gel was placed on the surface. A neutral (zero) electrode was first connected, and then the electrodes were placed on the middle parts of masseter muscles and the front parts of temporal muscles along the main direction of the muscle fibers. Thus, they correspond to the large part of the muscle, which is determined on maximum voluntary contraction (MVC). ${ }^{18}$

To measure the muscular activity, three tests were performed at the same session, with a rest period of about 2 minutes after each test. Each recording time was 10 seconds, and only 5 seconds were used to determine the EMG signal starting after 2.5 seconds of EMG start. The arithmetic mean was calculated for the three tests performed for each muscle (Fig. 2).

Hickman et al., reiterated that the best position for jaw during EMG is the position of MVC because it yields the best muscle activity. ${ }^{19}$ Hence, the EMG examination was performed to investigate the
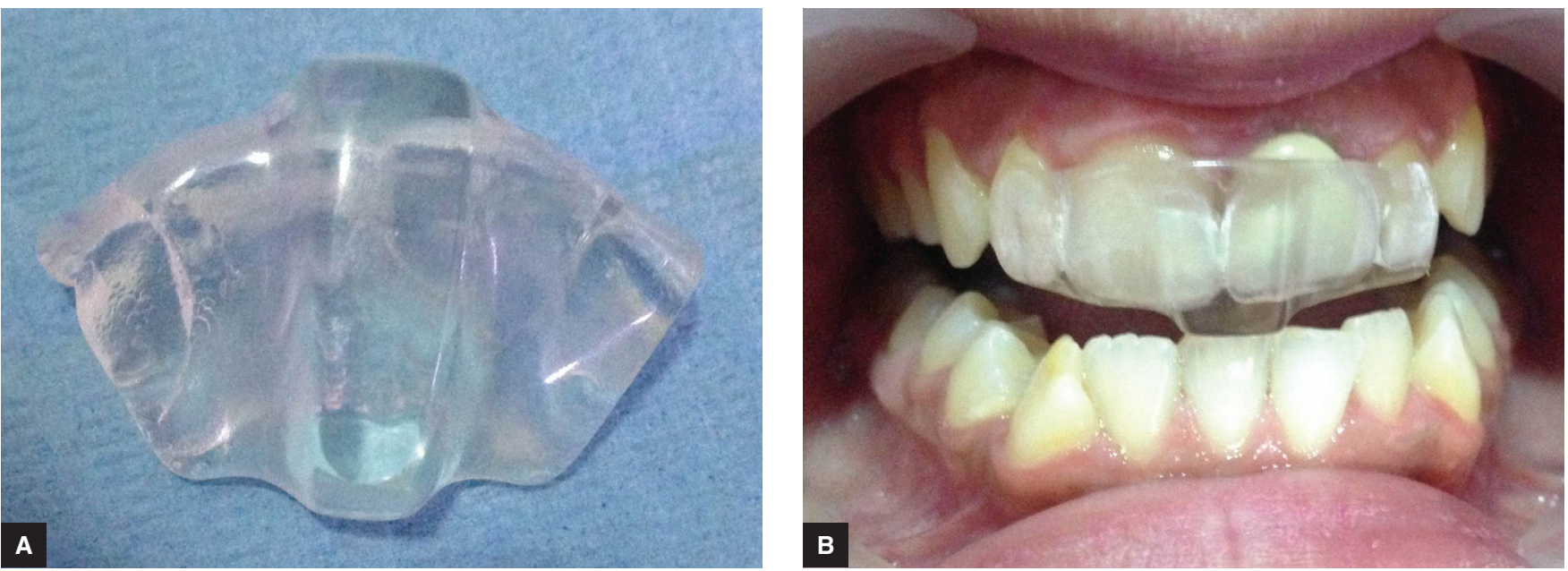

Figs 1 A and B: (A) The nociceptive trigeminal inhibition splint and (B) inserted nociceptive trigeminal inhibition splint 


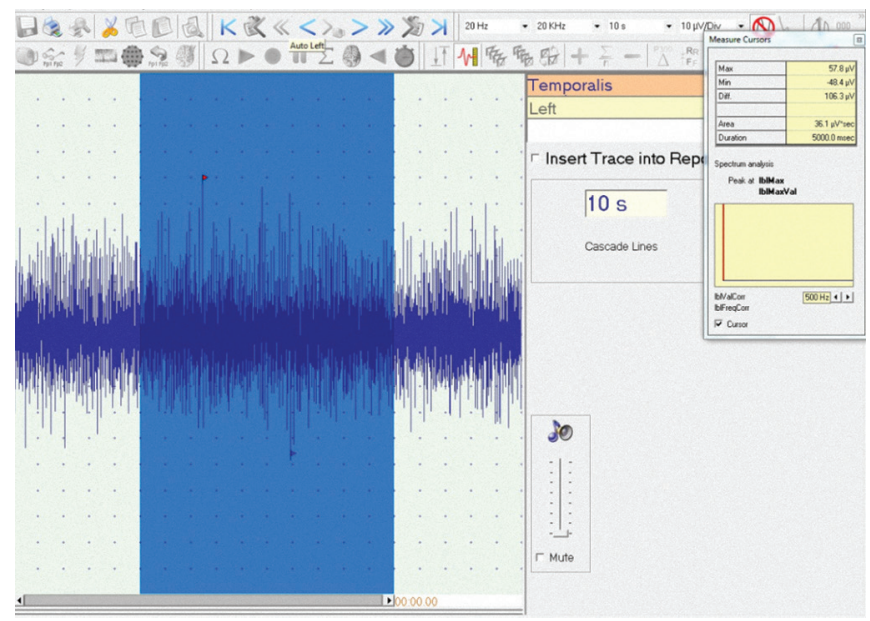

Fig. 2: EMG of the left temporal muscle. Max: the maximum potential. Min: the minimum potential. Diff: the difference between the highest and lowest potential $(\mu \mathrm{V})$

masseter and temporal muscles' activity in the position of MVC according to two time periods: before starting the treatment and after 6 months of treatment. These muscles were chosen because they are superficial, great, palpable, and accurately determinable. ${ }^{19}$

The patients' discomfort was assessed in the following times: the first day after starting the treatment, after 2 weeks, after 1 month, and after 6 months. The patients were asked to choose one of the four possible answers: not at all, lightly, yes to a specific degree, and yes to a clear degree.

All patients were treated and all measurements were made by one author "JK."

\section{Statistical Analysis}

Statistical analyses were performed using the Statistical Package for the Social Sciences (SPSS) software, (version 23; IBM Corporation, USA).
Data normality was assessed using the Shapiro-Wilk test. The paired-sample $t$ tests were employed to evaluate the intragroup electrical muscular activity changes. The Friedman test was employed to compare patients' discomfort degrees between the four study intervals, and the Wilcoxon matched-pairs signed-rank tests were used to fulfill the multiple comparisons.

\section{Results}

Table 1 shows that the values of the electrical muscular activity (microvolts) after the treatment were significantly smaller than those before the treatment, regardless of the muscles type or side $(p<0.001)$.

There were no statistically significant differences in the electrical muscular activity mean change between the masseter muscles $(-43.87 \pm 26.82)$ and the temporalis muscles $(-54.91 \pm 21.16)$ $(p=0.082)$, or between the right muscles $(-51.97 \pm 26.30)$ and the left muscles $(-46.81 \pm 22.90)(p=0.422)$, see Table 2 .

By comparing the frequency of patients' discomfort in the four study periods, statistically significant differences $(p<0.001)$ were observed, refer Table 3.

Table 4 displays that the degree of discomfort of the patients from the splints did not significantly change after 2 weeks of treatment $(p=0.180)$, while it gradually decreased in the following detecting periods $(p<0.01)$.

\section{Discussion}

Most of the TMDs are treated with different design splints because they are simple and conservative treatments and can easily relieve symptoms. ${ }^{20}$ There is scarcity of published clinical studies on the therapeutic efficacy of NTIS in patients with TMDs. Further, no clinical study examined the patients' discomfort degree when treated with it. The objective of this study was to evaluate the effects of NTIS in TMD patients, and to detect patients' discomfort degree to this splint.

The patients were asked to use the splint only during the night because it reduces muscle activity and bruxism, ${ }^{21}$ and to avoid any

Table 1: Pre and posttreatment differences in electrical activity mean in the study sample, according to the muscle type and side using the paired-samples $t$ test

\begin{tabular}{|c|c|c|c|c|c|c|c|c|c|}
\hline Muscles & Stage & $N$ & Mean & $S D$ & Min & Max & Diff & tvalue & $p$ value \\
\hline \multirow[t]{2}{*}{ Masseter (right and left) } & Pretreatment & 30 & 41.20 & 16.48 & 15.86 & 76.83 & -20.93 & -6.919 & $<0.001^{* * * *}$ \\
\hline & Posttreatment & 30 & 20.27 & 6.64 & 6.3 & 34.66 & & & \\
\hline \multirow[t]{2}{*}{ Temporal (right and left) } & Pretreatment & 30 & 51.50 & 15.15 & 21.63 & 87.5 & -29.46 & -10.572 & $<0.001^{* * * *}$ \\
\hline & Posttreatment & 30 & 22.04 & 10.38 & 7.8 & 44.83 & & & \\
\hline \multirow[t]{2}{*}{ Right (masseter and temporal) } & Pretreatment & 30 & 44.28 & 14.92 & 21.63 & 87.5 & -25.27 & -7.982 & $<0.001^{* * *}$ \\
\hline & Posttreatment & 30 & 19.01 & 7.98 & 6.3 & 34.66 & & & \\
\hline \multirow[t]{2}{*}{ Left (masseter and temporal) } & Pretreatment & 30 & 48.42 & 18.01 & 15.86 & 82.8 & -25.12 & -8.801 & $<0.001^{* * *}$ \\
\hline & Posttreatment & 30 & 23.30 & 8.96 & 14.6 & 44.83 & & & \\
\hline
\end{tabular}

$N$, number; SD, standard deviation; Min, minimal value; Max, maximal value; Diff, difference; $t$ value, test statistic; $p$ value, significance level; ${ }^{* * * *} p<0.001$

Table 2: Differences in the electrical muscular activity between pre and posttreatment values according to the muscle type and side using the paired-sample $t$ test

\begin{tabular}{|c|c|c|c|c|c|c|c|c|}
\hline Muscles & $N$ & Mean & $S D$ & Min & Max & Diff. & $t$ value & $p$ value \\
\hline Masseter & 30 & -43.87 & 26.82 & -77.3 & 0 & 11.04 & 1.771 & 0.082 \\
\hline Temporal & 30 & -54.91 & 21.16 & -88.3 & -10.0 & & & \\
\hline Right & 30 & -51.97 & 26.30 & -88.2 & -2.1 & -5.15 & -0.809 & 0.422 \\
\hline Left & 30 & -46.81 & 22.90 & -72.6 & 0 & & & \\
\hline
\end{tabular}

$N$, number; SD, standard deviation; Min, minimal value; Max, maximal value; Diff., difference; $t$ value, test statistic; $p$ value, significance level 
Table 3: Differences in frequency of patients' discomfort between the four study periods using the Friedman test

\begin{tabular}{llll}
\hline Study periods & Mean & $x^{2}$ value & $p$ value \\
\hline After 1 day & 2.93 & 27.383 & $<0.001^{* * *}$ \\
2 weeks later & 3.33 & & \\
1 month later & 2.20 & & \\
6 months later & 1.53 & & \\
\hline$x^{2}$ value, test statistic $p$ value significance level ***p<0.001 &
\end{tabular}

Table 4: The multiple comparisons of frequency of the degrees of patients' discomfort using the Wilcoxon test

\begin{tabular}{llcc}
\hline Study periods & & z value & $p$ value \\
\hline 1 day & 2 weeks & -1.342 & 0.180 \\
& 1 month & -2.333 & $0.020^{*}$ \\
& 6 months & -2.919 & $0.004^{* *}$ \\
2 weeks & 1 month & -2.887 & $0.004^{* *}$ \\
& 6 months & -3.418 & $<0.001^{* * *}$ \\
1 month & 6 months & -2.449 & $0.014^{*}$ \\
\hline
\end{tabular}

$z$ value, test statistic; $p$ value, significance level; ${ }^{*} p<0.05,{ }^{* *} p<0.01$, ${ }^{* * *} p<0.001$

changes in occlusion. These occlusion changes may occur if there is no occlusal contact in the posterior region for 8 continuous days. ${ }^{22}$

This study showed a significant reduction in muscles' activity after treatment for both the masseter and temporalis muscles in both right and left sides. This decrease could be ascribed to the separation of occlusion between the posterior teeth which leads to the removal of the posterior occlusion interferences; this, in turn, decreases masticatory muscles' activity. ${ }^{7}$

The findings of this study are consistent with those of BaadHansen et al., who designed their study to address the question about the EMG changes during sleep. They noticed that there was a significant reduction in the activity of the masseter muscle during the use of NTIS in 10 patients (age: 23-39 years) with toothgrinding during sleep associated with TMDs. ${ }^{23}$ The current results also accord with those of Becker et al., who measured the effect of a prefabricated anterior bite stop on the activity of the temporalis and masseter muscles and noticed a reduction in the muscles' activities in the intercuspal position and during the functional movements. ${ }^{24}$

On the contrary, the findings of this study disagree with those of Dalewski et al., who did not notice a significant effect of NTIS on the activity of the masseter and temporalis muscles in patients with bruxism. ${ }^{9}$ This difference may be attributed to the study period which was only 30 days in their study, while it was 6 months in the current one.

This study showed that there were no significant differences in the mean change in muscles' activity between the masseter and temporalis muscles, or between the right and left sides. This may reveal that the splint resulted in an equal distribution of the forces on these two muscles. Dalewski et al., did not find significant differences in the muscle activity between the masseter and temporalis muscles, or between the right and left sides after 30 days of treatment with NTIS in patients with bruxism, keeping in mind that they did not observe any significant effects of this splint on these muscles. ${ }^{9}$

The current study showed a decrease in the degree of discomfort resulting from NTIS as treatment time passes on. During the first 2 weeks, there was no improvement in the degree of discomfort, and then it significantly decreased as the study time goes by. This discomfort can be attributed to the oral touching stimuli caused by the presence of a foreign body in the mouth. These results agree with those of Conti et al., whose patients expressed discomfort from using NTIS during the first 15 days. ${ }^{25}$

\section{Conclusions}

- The use of NTIS associates with a significant reduction in the masseter and temporalis muscles' activity.

- There are no significant differences between the masseter and temporalis muscles' activity mean change resulting from the treatment with NTIS.

- The degree of discomfort from NTIS gradually decreases after 2 weeks, 1 month, and 6 months of treatment.

\section{Clinical Significance}

The NTIS is an effective therapeutic approach for TMD patients of muscular origin. Furthermore, EMG is an important method guiding clinicians on the opportunity to prescribe new different therapeutic means.

\section{References}

1. Costen JB. A syndrome of ear and sinus symptoms dependent upon disturbed function of the temporo-mandibular joint. Ann Otol Rhinol Laryngol 1934 Mar;43(1):1-15.

2. Dworkin SF. Temporomandibular disorder (TMD) pain-related disability found related to depression, nonspecific physical symptoms, and pain duration at 3 international sites. J Evid Based Dent Pract 2011 Sep;11(3):143-144. DOI: 10.1016/j.jebdp.2011.06.002.

3. McNamara Jr JA, Seligman DA, et al. Occlusion, orthodontic treatment, and temporomandibular disorders: a review. J Orofac Pain 1995 Winter;9(1):73-90.

4. Coskun Benlidayi I, Salimov F, et al. Kinesio taping for temporomandibular disorders: single-blind, randomized, controlled trial of effectiveness. J Back Musculoskelet Rehabil 2016 Apr;29(2): 373-380. DOI: 10.3233/BMR-160683.

5. Ferreira FM, Cézar Simamoto-Júnior $P$, et al. Effect of occlusal splints on the stress distribution on the temporomandibular joint disc. Braz Dent J 2017 May-Jun;28(3):324-329. DOI: 10.1590/01036440201601459.

6. Ommerborn MA, Taghavi J, et al. Therapies most frequently used for the management of bruxism by a sample of German dentists. J Prosthet Dent 2011 Mar;105(3):194-202. DOI: 10.1016/S00223913(11)60029-2.

7. Klasser GD, Greene CS. Oral appliances in the management of temporomandibular disorders. Oral Surg Oral Med Oral Pathol Oral Radiol Endod 2009Feb;107(2):212-223. DOI: 10.1016/j.tripleo.2008.10.007.

8. Gray R, Davies S, et al. A clinical approach to temporomandibular disorders. 6 splint therapy. Br Dent J 1994 Aug;177(4):135-142.

9. Dalewski $B$, Chruściel-Nogalska $M$, et al. Occlusal splint versus modified nociceptive trigeminal inhibition splint in bruxism therapy: a randomized, controlled trial using surface electromyography. Aust Dent J 2015 Dec;60(4):445-454. DOI: 10.1111/adj.12259.

10. Conti PCR, De Alencar E, et al. Behavioural changes and occlusal splints are effective in the management of masticatory myofascial pain: a short-term evaluation. J Oral Rehabil 2012 Oct;39(10):754-760. DOI: $10.1111 / \mathrm{j} .1365-2842.2012 .02327 . x$.

11. Stapelmann H, Türp JC. The NTI-tss device for the therapy of bruxism, temporomandibular disorders, and headache-where do we stand? A qualitative systematic review of the literature. BMC Oral Health 2008 Jul;8(1):22. DOI: 10.1186/1472-6831-8-22.

12. Choi K-H, Kwon OS, et al. Development of electromyographic indicators for the diagnosis of temporomandibular disorders: a protocol for an assessor-blinded cross-sectional study. Integr Med Res 2017 Mar;6(1):97-104. DOI: 10.1016/j.imr.2017.01.003. 
13. Shankland WE. Nociceptive trigeminal inhibition-tension suppression system: a method of preventing migraine and tension headaches. Compend Contin Educ Dent 2001 Dec;22(12):1075-1080, 1082; quiz 1084.

14. Bumann A, Lotzmann U. Color atlas of dental medicine: tmj disorders and orofacial pain, the role of dentistry in a multidisciplinary diagnostic approach. 1st ed. New York: Thieme, 2002; p. 53.

15. Magnusson T, Adiels A-M, et al. Treatment effect on signs and symptoms of temporomandibular disorders-comparison between stabilisation splint and a new type of splint (NTI). A pilot study. Swed Dent J 2004;28(1):11-20.

16. Abou-Atme YS, Chedid N, et al. Clinical measurement of normal maximum mouth opening in children. Cranio 2008 Jul;26(3):191-196. DOI: $10.1179 / \mathrm{crn} .2008 .025$.

17. Koc D, Dogan A, et al. Effects of increasing the jaw opening on the maximum bite force and electromyographic activities of jaw muscles. J Dent Sci 2012 Mar;7(1):14-19.

18. Lodetti G, Marano G, et al. Surface electromyography and magnetic resonance imaging of the masticatory muscles in patients with arthrogenous temporomandibular disorders. Oral Surg Oral Med Oral Pathol Oral Radiol 2014 Aug;118(2):248-256. DOI: 10.1016/ j.00oo.2014.05.005.
19. Hickman DM, Cramer R, et al. The effect of four jaw relations on electromyographic activity in human masticatory muscles. Arch Oral Biol 1993 Mar;38(3):261-264.

20. Forssell H, Kalso E. Application of principles of evidence-based medicine to occlusal treatment for temporomandibular disorders: are there lessons to be learned? J Orofac Pain 2004 Winter;18(1):9-22.

21. Okeson JP. The effects of hard and soft occlusal splints on nocturnal bruxism. J Am Dent Assoc 1987 Jun;114(6):788-791.

22. Aksakalli $S$, Temucin $F$, et al. Effectiveness of two different splints to treat temporomandibular disorders. J Orofac Orthop 2015 Jul;76(4):318-327. DOI: 10.1007/s00056-015-0294-4.

23. Baad-Hansen $L$, Jadidi $F$, et al. Effect of a nociceptive trigeminal inhibitory splint on electromyographic activity in jaw closing muscles during sleep. J Oral Rehabil 2007 Feb;34(2):105-111. DOI: 10.1111/ j.1365-2842.2006.01717.x.

24. Becker I, Tarantola G, et al. Effect of a prefabricated anterior bite stop on electromyographic activity of masticatory muscles. J Prosthet Dent 1999;82(1):22-26.

25. Conti PCR, Corrêa AS, et al.Management of painful temporomandibular joint clicking with different intraoral devices and counseling: a controlled study. J Appl Oral Sci 2015;23(5):529-535. DOI: 10.1590/ 1678-775720140438. 